\title{
Alzheimer, \\ la vie, la mort, \\ la reconnaissance
}

\section{Michel Malherbe}

\section{Alice Quercy}

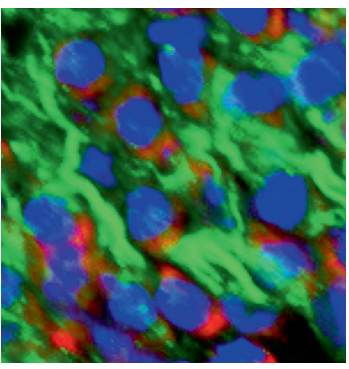

CNRS UMR 7219, laboratoire SPHERE (Sciences, Philosophie, Histoire), bâtiment Condorcet, case 7093, 5, rue Thomas Mann 75205 Paris Cedex 13, France. alice.quercy@gmail.com

Retraçant la relation entre lui-même et son épouse, plus que la progression de la maladie elle-même, Malherbe s'attache à montrer ce qu'il appelle «l'autre versant». Celui que le bien portant ne voit pas forcément. Celui que le commun néglige peut-être. Dans cette relation, un faible et un fort apparaissent. Le malade, démis de tout tion du philosophe autant qu'elle aiguise la douleur du mari qui voit sa femme dépérir d'Alzheimer [1]. Qui est « encore » Annie?

L'auteur, Michel Malherbe ${ }^{1}$, est un philosophe spécialisé dans l'empirisme anglo-saxon ${ }^{2}$. La maladie de son épouse le conduit à travailler sur un mode personnel, et assumé comme tel, des questions philosophiques classiques sur les idées de personne et d'identité. II adopte pour cela une philosophie levinasienne ${ }^{3}$ [2], imprégnée de questions sur l'éthique et le rapport à autrui. Lorsque sa femme tombe malade, de la pathologie d'Alzheimer, Malherbe décide ainsi de témoigner dans un livre original où se mêlent questions de philosophe et observations douloureuses de la réalité.

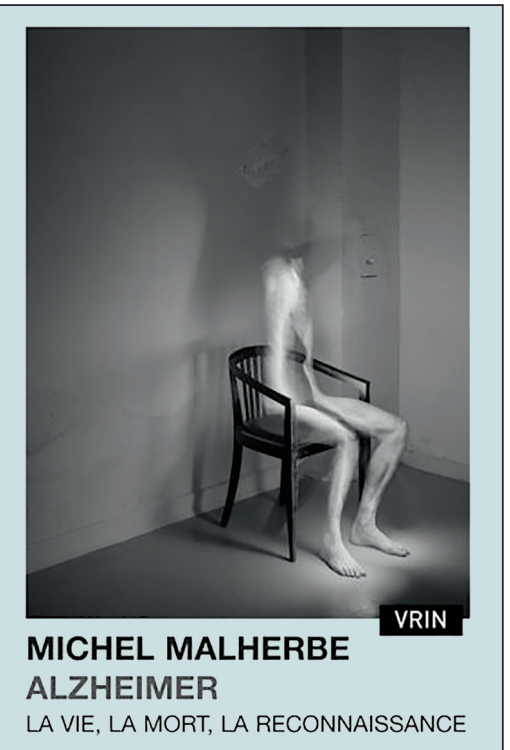
pouvoir de communication et d'action « est donné » au plus fort, le «bien portant ». Pour autant, cette relation n'est pas figée. Derrière l'aidant ${ }^{4}$, se cache un homme meurtri qui ne sait plus «qui il aide», «qui il assiste». Nous observons une relation dénuée de sens, une relation où la souffrance est parfois même inversée : Le malade souffre-t-il autant «psychiquement », que son proche qui le voit s'affaiblir de jour en jour?

Dans cette relation de souffrance, où le « sens » peu à peu disparaît, la maladie, elle, s'installe, insidieusement. Désignée comme le serpent vicieux, la maladie est la représentation «du mal » d'une époque. Et l'interrogation philosophique du narrateur introduit clairement cette notion « Si la maladie est le diable, je ne suis pas le Dieu ». Preuve que la maladie est un phénomène que l'auteur qualifie de narrateur, surtout quand la philosophie et la médecine sont confrontées à leurs propres impasses face à cette maladie dévastatrice et déroutante.

\footnotetext{
${ }^{1}$ Michel Malherbe est un philosophe français né en 1941. II a traduit plusieurs auteurs anglais d'ouvrages philosophiques dont Bacon, Locke et Hume.

${ }^{2}$ L'empirisme anglo-saxon est une théorie de la connaissance fondée sur l'expérience par opposition à des théories innéistes.

${ }^{3}$ Emmanuel Levinas (1906-1995) est l'un des premiers penseurs à faire de l'existence un problème philosophique.
} «totalitaire » car s'insinuant dans tous le(s) corps, dans tous les esprits. Incontrôlable, elle devient maître de la totalité d'un univers : celui du malade et de son entourage.

Cependant, si la maladie est plus forte actuellement que tous remèdes ou toute assistance, elle ne doit pas rester une simple interrogation, mais apparaître comme l'objet d'un véritable combat que chacun doit ${ }^{4}$ Une personne est considérée comme « aidant » lorsqu'elle s'occupe quotidiennement d'un proche
dépendant. 
mener comme un combat à gagner: sans quoi le travail philosophique serait stérile pour l'auteur.

Alors que la communication devient peu à peu impossible entre le malade et le proche, Malherbe s'intéresse particulièrement à la question de la reconnaissance.

On me demande: «Votre épouse vous reconnaît-elle?» Je réponds: «Peut être. Je ne sais. Mais la vraie question est autre, est-ce que moi je la reconnais?»

Si le mari observe qu'il ne reconnaît pas/plus sa femme, il s'interroge ainsi sur «cette présence qui est absence, ou absence qui est présence ».

Qui se cache derrière la maladie? La mort ou la vie ? Un être humain ou un être inhumain? Y a-t-il encore une humanité dans un homme sans conscience?

À partir de ces questions, Malherbe entame sa réflexion philosophique. II va d'abord chercher à définir ce qu'est l'être humain, ce que pourraient être les critères déterminant l'être humain. L'objectif étant de répondre à une question «Qui est l'être malade? Comment appréhender, vivre et maintenir une relation avec celui-ci? ». Ici Malherbe répond au vœu précédemment souhaité de mettre en œuvre sa philosophie au service de la cause concrète «Alzheimer ».

Les philosophes sont alors mis à contribution. La pluralité de leurs visions enrichit le propos de l'auteur sur ce que peut être l'être humain. Par exemple, pour Locke [3] (1632-1704), la conscience semble être un critère de définition indispensable pour l'identité de la personne. Pour Kant [4] (1724-1804), le «je pense » est la condition de toute existence dans le monde, car il dispose de la rationalité. La méthode Kantienne appliquée à la maladie d'Alzheimer consisterait ainsi à considérer tout être comme son semblable «parce que » c'est un impératif moral (catégorique) de le considérer en tant que tel. Tout acte (maxime) devant pouvoir s'appliquer universellement, l'acte appliqué au malade doit pouvoir s'appliquer à tout être rationnel. Ainsi, la volonté entreprise dans la mise en place de soins et dans l'assistance doit correspondre à cette rationalité qui doit s'approcher de l'universalité.

Malherbe ne se contente pas de citer ces deux auteurs mais en fait un usage critique. Car la maladie d'Alzheimer défie la philosophie dans l'ensemble de ses termes, de ses définitions, de ses auteurs. Cela est indiqué dans le prologue et répété tout au long de ce récit : la maladie d'Alzheimer laisse impuissante la philosophie. D'où la genèse répétée de ce livre: Malherbe nous propose un récit personnel sous forme de quête philosophique.

En l'opposant aux deux auteurs précédemment cités, Malherbe s'intéresse alors à Max Scheler ${ }^{5}$, philosophe allemand qui a notamment formulé une morale de la «sympathie » [5].

Scheler a une vision « relationnelle » de l'identité : «La rencontre de l'autre [...] ne requiert que ceci : faire advenir l'autre rencontré comme sens et valeur »[6]. Selon Scheler, le malade res-

\footnotetext{
${ }^{5}$ Max Scheler (1874-1928) est un philosophe et sociologue allemand.
}

terait donc un être humain qui garderait tout son sens et sa valeur. Et «La femme aimée » resterait alors aux yeux de son mari, «unique ». Sa méthode semble donc plus porteuse de sens pour l'auteur, car plus proche des sentiments du «narrateur». Finalement, Malherbe défend ici une vision «souple» de l'identité, où l'individu se construit dans sa relation à autrui. L'aidant est alors invité à apprécier l'autre «qui est devenu autre ». Cet «Autre qui est autre», répété inlassablement, doit être compris dans une altérité qui n'est pas fondée communément sur la ressemblance mais directement dans une altérité méconnue mais comprise dans la nature humaine. On retrouve ici I'influence de Levinas sur Malherbe.

Sur une autre échelle, la reconnaissance de l'individu reste une question très concrète. Comme le dit le narrateur : «la maladie d'Alzheimer est une maladie terriblement réelle ». Donc à la question d'ordre philosophique de ce qu'est l'être humain dans sa relation à l'autre, l'auteur recherche comment maintenir la relation face au mal physique, observable.

Premièrement, l'auteur s'intéresse à cette ambivalence entre le corps et l'esprit. Le corps et l'esprit ne font-ils qu'un ? Où sont-ils deux « choses » séparées, distinctes? La particularité de la maladie d'Alzheimer est qu'elle combine à la fois la dégénérescence physique et mentale. Défiant notre capacité scientifique à la comprendre, la maladie soulève ainsi plusieurs questions. Qui est la cause, qui est la conséquence, quels sont les liens?

Si on induit l'idée que la maladie est entièrement physique, comment comprendre l'absence de «réaction » sentimentale, comment accepter que le patient Alzheimer ait fait une fuite, pour la dixième fois, de l'établissement sans en être totalement conscient? Cette solution paraît insurmontable psychiquement pour l'aidant. Et destructrice pour la relation.

Au contraire, pour l'auteur, le corps et l'esprit sont distincts mais ils doivent cependant être reconnus comme dépendants l'un de l'autre. Ainsi «la reconnaissance morale de la personne passe par la connaissance qu'il faut acquérir de sa réalité physique »; nous devons accepter l'individu tel quel, dans son (in-)activité physique.

La réalité «physique » s'intègre aussi dans la réalité du « quotidien ». Accompagnement ou assistance, vie « artificiellement maintenue à la maison » ou « artificiellement entretenue à l'institut » ? L'auteur/narrateur témoigne des insuffisances de la connaissance de la maladie.

Il évoque ainsi la douloureuse expérience de « l'institut », cette maison médicalisée pour les patients atteints 
d'Alzheimer qui délaisse la relation d'avant pour entrer dans un après. Cet après semble lui être tout à fait faux. On ressent une contradiction personnelle non résolue quant à la volonté de maintenir un semblant d'humanité face à des personnes non « responsables », contre l'hypocrisie délibérée (mais vécue comme nécessaire) de chaque aidant, chaque infirmier pour assurer le soin de chacun. Des exemples en témoignent, notamment avec l'étude du lien dépendance/compétence : la « décision » des soignants de donner le goûter puisque les patients sont dans l'incapacité de l' « organiser ». Mais la volonté de «laisser le choix », la compétence aux patients de choisir la boisson. Cette «compétence » laissée aux patients peut être «feinte».

Ces questions pratiques mettent en évidence certaines limites de la loi Kouchner du 4 mars $2002^{6}$ sur le consentement obligatoire. D'une part, comment maintenir ce consentement lorsque la personne n'est plus capable, rationnellement, de comprendre et d'accepter la maladie qui l'affecte? D'autre part, comment substituer ce principe par le biais de la tutelle en ne rendant pas l'autre « irresponsable » et donc « non semblable » à moi qui suit «le bien portant »?

Par ailleurs, l'acte de reconnaissance de l'aidant n'est pleinement « réalisé » que s'il y a une co-reconnaissance de la part du patient: la cuillère portée au malade n'est possible que si le patient réussit à enclencher l'acte d'ouvrir la bouche. Cet acte de co-reconnaissance, nécessaire à l'accomplissement total du soin, reste problématique. De sorte que, selon l'auteur, la relation avec un malade d'Alzheimer, fut-il son conjoint, relève d' « une reconnaissance incomplète ». Sauf si, l'«attachement pour la personne aimée», ou l'insistance acharnée du personnel médical résistent à la dégénérescence de l'être et à la violence persistante de la maladie.
Ce récit de Malherbe combinant témoignage douloureux et réflexion philosophique est ainsi une quête harassante où ni le philosophe ni le mari ne trouvent finalement de solutions pour combattre effectivement ce mal. Fabrice Gzil [7], responsable du pôle Études et recherche de la Fondation Médéric Alzheimer, est le premier philosophe en France à s'être intéressé à la prise en charge et l'amélioration du vécu de ces patients et de leur entourage. Il propose des solutions alternatives qui pourront alimenter le propos de Malherbe dans une perspective plus politique et sociale. $\diamond$

Alzheimer, life, death, recognition

\section{LIENS D'INTÉRÊT}

L'auteur déclare n'avoir aucun lien d'intérêt concernant les données publiées dans cet article.

\section{RÉFÉRENCES}

1. Malherbe M. Alzheimer, la vie, la mort, la reconnaissance. Paris : Vrin, 2015, $298 \mathrm{p}$.

2. Levinas $\varepsilon$. Totalité et infini, essai sur l'extériorité, $4^{\text {e }}$ ed. La Haye : Martinus Nijhoff, $1980: 161-225$.

3. Locke J. Essai. Op. cit. III 279 sq.

4. Kant $\varepsilon$. Fondements de la métaphysique des mœurs. Traduction V. Delbos. Paris : Vrin, 1993, $74 \mathrm{p}$.

5. Scheler M. Nature et formes de la sympathie. Traduction M. Lefebvre. Paris : Payot, $2003: 476 \mathrm{p}$.

6. Scheler M. Le formalisme en éthique et l'éthique matériale des valeurs. Essai nouveau pour fonder un personnalisme éthique (1913-1916). Traduction M. de Gandillac. Paris : Gallimard, 1955.

7. Gzil F, Hirsch $\varepsilon$. Alzheimer, éthique et société. Toulouse : Érès, Poche-Espace éthique, 2012, $686 \mathrm{p}$.

\footnotetext{
${ }^{6}$ Loi relative aux droits des malades et à la qualité du système de santé.
}

\section{TIRÉS À PART}

\section{A. Quercy}

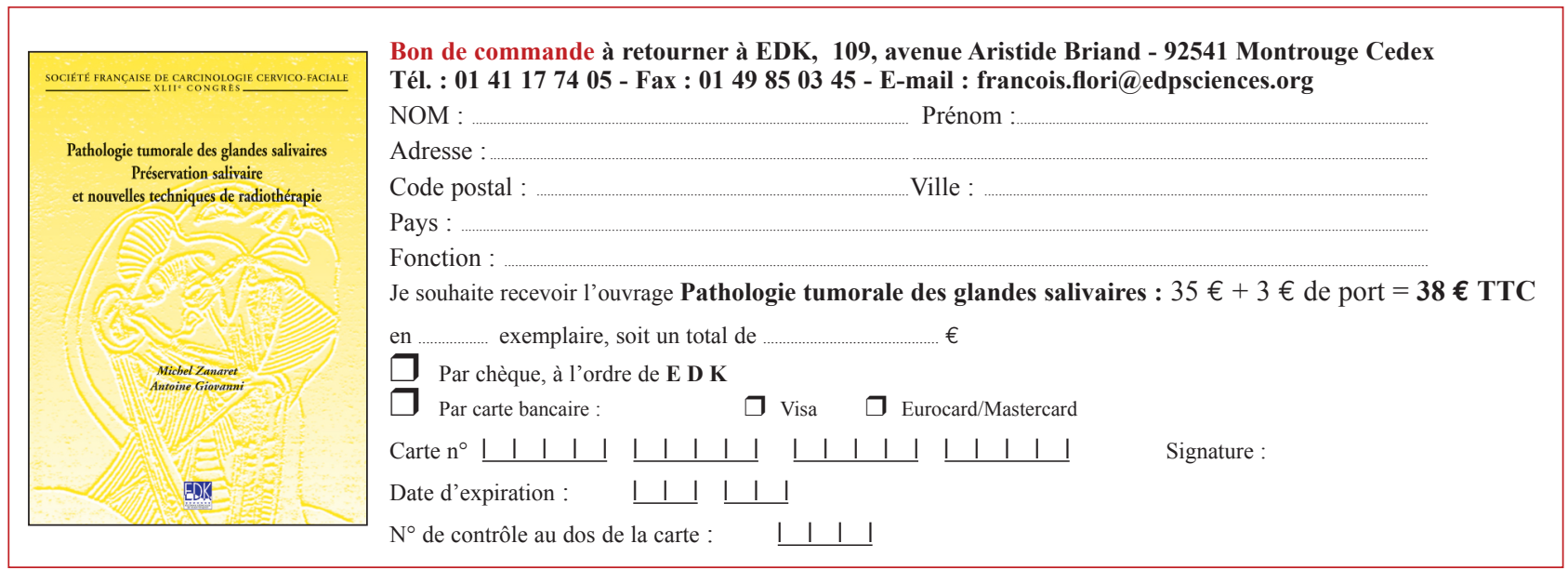

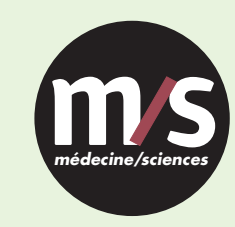

Tarifs d'abonnement $\mathrm{m} / \mathrm{s}-2016$

Abonnez-vous

à médecine/sciences
$>$ Grâce à $m / s$, vivez en direct les progrès des sciences biologiques et médicales

Bulletin d'abonnement page 724 dans ce numéro de $\mathrm{m} / \mathrm{s}$

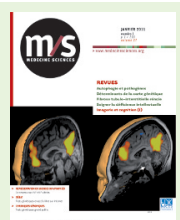

